\title{
Gait Recognition for Human Identification Using Fourier Descriptor and Anatomical Landmarks
}

\author{
Mridul Ghosh \\ Department of Computer Science and Engineering, Supreme Knowledge Foundation Groups Of Institutions, Hooghly, \\ India \\ mridulxyz@gmail.com
}

Debotosh Bhattacharjee

Department of Computer Science and Engineering, Jadavpur University, Kolkata, India debotosh@ieee.org

\begin{abstract}
This paper presents a gait recognition method which is based on spatio-temporal movement characteristics of human subject with respect to surveillance camera. Different measures, like leg rise from ground (LRFG), the angles created between the legs with the centre of Mass (ABLC), angles created between the Centre of Mass Knee and Ankle with the (CKA), angles created between Centre of Mass, Wrist and knee $(\mathrm{CWK})$, the distances between the control points and centre of Mass (DCC) have been taken as different features. Fourier descriptor has been used for shape extraction of individual frames of a subject. Statistical approach has been used for recognition of individuals based on the $\mathrm{n}$ feature vectors, each of size 23(collected from LRFG, ABLCs, CKA, CWK and DCCs) for each video frame. It has been found that recognition result of our approach is encouraging with compared to other recent methods.
\end{abstract}

Index Terms - Gait Recognition, Fourier Descriptor, centre of Mass, Mahalanobis Distance.

\section{INTRODUCTION}

Physical biometric traits like face, fingerprint and iris are typically used in applications to identify human beings. Recent research has demonstrated the possible ancillary information such as gender, height, weight, age and ethnicity to improve the recognition accuracy of biometric systems.

Gait is the systematic study of human walking styles and in due course of time it has drawn lots of attention of people. For example, Borelli [1], an Italian Scientist, in $17^{\text {th }}$ Century, pioneered the animal gait movement (locomotion). Braune [2] in the year of 1890 did a tremendous work on biomechanics using lithographic cross section of human body.

Human gait shows distinct characteristic of person and also the walker's physical and psychological status. Research on gait analysis can be expressed on medical perspective. Murray [3] used gait to classify the pathologically abnormal patients into different group for appropriate treatment. This classification was achieved by comparing a patients' gait patterns with normal gait patterns obtained from a control group.

Because of its potential for human identification at distance human gait recognition is developing tremendously in terms of computer vision research. Since it is difficult to identify person from pictures taken from a distance by some other biometric, gait has made it possible by taking a person's image in different sequences.

Different methods and approaches have been proposed to identify human beings throughout more than a decade.

Jiwen Lu and Erhu Zhang [4] proposed a method for gait recognition on the basis of human silhouettes using multiple feature representations and Independent Component Analysis (ICA).

Considering multiple features and fusing them and classify on the basis of Genetic Fuzzy Support Vector Machine (GFSVM), good recognition result has been obtained. In their proposed method they have considered three view fusions, i.e. perpendicularly, along and oblique with the direction of human walking.

Lee and et. al [5] proposed a method that attempted to divide the human body into components that are treated separately. In their work, a silhouette is represented by seven ellipses and by using Fourier transform they extract features from the change in magnitude and phase components and discrimination power from these components during walking of the subject. These features were used for person identification and gender classification and for relatively small dataset it produced good results.

Cunado et al. [6] proposed an approach where gait signature is extracted from a sequence of walking using the Fourier series to represent the motion of the upper leg. K-nearest neighbor classification rule was applied to the Fourier components of the motion of the upper leg. By the phase-weighted Fourier magnitude information when compared to the usage of the magnitude information enhanced classification rate is obtained.

By the analysis of image sequence, visual analysis of human motion [7] [8] detection, tracking and identification of any object has become possible. Over 
the past few years, the combination of biometrics and human motion has become a popular research. The vision based human identification at a distance has gained wider interest from the computer vision community. For automated person identification system in visual surveillance and monitoring application in security purpose such as banks, parking lots, airports and railway station etc this interest is strongly motivated.

\section{Contributions of this Paper}

Here, Fourier Descriptor has been applied to for shape extraction and analytical landmarks have been considered for control point selection.

Different angles, like angle created between the Centre of Mass, Knee and Ankle (CKA), angle created between Centre of Mass, Wrist and knee (CWK) have been measured along with angles i.e., DCC and Distances between the Centre of mass and the Control points i.e. ABLC have been calculated.

Section 2 describes feature extraction technique,

Experiments conducted for this work along with results are described in section 3 and section 4 concludes this work.

\section{FEATURE EXTRACTION}

For any kind of recognition concern the feature extraction is a vital and major part. Before approaching the feature extraction stage, it is needed to collect the video sequences of different persons and dividing the video sequences in fixed time interval the static images of every moment of walk is taken in such a way that the entire gait movement cycle can be obtained and by the following procedures they are converted to binarized silhouette images to simplify our work. Features are extraction from these silhouettes has been described below.

\subsection{Object extraction}

Considering that the object is moving and camera is static, the video sequences have been taken and the snapshots have been taken in such a time interval that the gait signatures are fully realized.

The first and foremost thing is to subtract the background from the entire image to have the foreground object or the subject under concern.

Based on background subtraction and silhouette correlation the extraction and tracking of moving silhouettes from the background in each image-frame the detection and tracking algorithm is used.

By LMedS (Least Median of Square) [9] method background image can be generated from a small portion of image sequence.

Siegel's estimator [9] for LMedS is defined as follows: For any m observations $\left(\mathrm{x}_{\mathrm{i}_{1}}, \mathrm{y}_{\mathrm{i}_{1}}\right), \ldots .,\left(\mathrm{x}_{i_{m}}, \mathrm{y}_{i_{m}}\right)$ which determine a unique parameter vector, the $\mathrm{jth}$ coordinate of this vector, denoted by $\theta_{j}\left(i_{1}, \ldots \ldots, i_{m}\right)$. The repeated median is then defined coordinate-wise as

$$
\hat{\theta_{\mathrm{j}}}=\operatorname{med}_{i_{1}}\left(\ldots . .\left(\operatorname{med}_{i_{p-1}}\left(\operatorname{med}_{i_{m}} \theta_{\mathrm{j}}\left(\mathrm{i}_{1}, \ldots, \mathrm{i}_{\mathrm{m}}\right)\right)\right) \ldots\right) \ldots \ldots(1)
$$

Let I represent a sequence including $\mathrm{N}$ images. The background $\beta(\mathrm{x}, \mathrm{y})$ can be computed as given in [9], by

$$
\beta(\mathrm{x}, \mathrm{y})=\min _{b}\left\{\operatorname{med}_{\mathrm{f}}\left(\mathrm{I}^{\mathrm{f}}(\mathrm{x}, \mathrm{y})-\mathrm{b}\right)^{2}\right\} .
$$

Where, $b=$ background brightness value at pixel

location $(\mathrm{x}, \mathrm{y})$. med $=$ median value

$\mathrm{f}=$ frame index from 1 to $\mathrm{N}$

It is difficult to select suitable threshold for binarization since the brightness of the entire sequence of gait cycle may not be same throughout, i.e. it may vary on time, and the brightness change can be obtained through differencing between the background and the current image and especially in case of low contrast image as the brightness change is too low to distinguish between object from noise. This problem can be solved by the following function which has been used to perform differencing [10] indirectly

$$
m(\alpha, \beta)=1-\frac{2 \sqrt{(\alpha(x, y)+1)(\beta(x, y)+1)}}{(\alpha(x, y)+1)+(\beta(x, y)+1)} \cdot \frac{2 \sqrt{(\eta-\alpha(x, y))(\eta-\beta(x, y))}}{(\eta-\alpha(x, y))+(\eta-\beta(x, y))}
$$

Where, $\alpha(\mathrm{x}, \mathrm{y})$ is the brightness of current image at pixel position $(\mathrm{x}, \mathrm{y}), \beta(\mathrm{x}, \mathrm{y})$ is the background at pixel position (x,y) of current image, $\eta$ is the maximum intensity value i.e. for gray scale it is $255,0<=\alpha(\mathrm{x}, \mathrm{y})$, $\beta(\mathrm{x}, \mathrm{y})<=\eta$ and $0<=m(\alpha, \beta)<1$.

Erosion and dilation operations are generally used to fill small holes inside the extracted silhouette to remove noise as much as possible

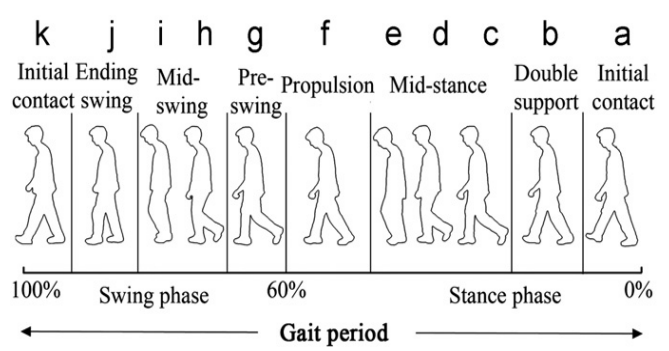

Fig.1. The specific phases of gait period (a)-(k): stance phase (a)-(f) comprising about $60 \%$ of the gait period; and swing phase $(\mathrm{g})-(\mathrm{j})$

There are two phases of walking, the Stance phase and Swing phase. Swing phase occurs when one leg will be in air and in the Stance phase both the legs will be on ground. It has been observed that $60 \%$ of walk is in Swing phase and $40 \%$ is in Stance phase. There are ten 
sub-phases in between Swing and Stance which can be seen from the figure1.

\subsection{Fourier Descriptor for shape extraction-}

The Fourier descriptor helps in describing the boundary of the object effectively. The boundary point $\mathrm{k}$ can be expressed as complex function

$$
\mathrm{S}(\mathrm{k})=\mathrm{X}(\mathrm{k})+\mathrm{j} \mathrm{Y}(\mathrm{k})
$$

The procedure of obtaining Fourier descriptor [11] is illustrated as follows:

i) Take $\mathrm{N}$ digital points of the boundary, where $\mathrm{N}$ represents the length of the closed curve.

ii) Apply the Fourier transform to the boundary points.

$$
\mathrm{S}(\mathrm{u})=\frac{1}{N} \sum_{k=0}^{N-1} T(k) e^{\frac{j 2 \Pi u x}{N}} \text {,for } \mathrm{k}=0,1,2, \ldots \mathrm{N}-1
$$

iii) The inverse transform can be applied to obtain the original points

$$
\mathrm{T}(\mathrm{k})=\frac{1}{N} \sum_{u=0}^{N-1} S(u) e^{\frac{j 2 \Pi u x}{N}} \quad \text {, for } \mathrm{u}=0,, 1,2, \ldots \mathrm{N}-1
$$

The coefficients $\mathrm{S}(\mathrm{u})$ are called Fourier descriptors. They represent the discrete contour of a shape in the Fourier domain.

Scale invariance of Fourier descriptors can be achieved by dividing all Fourier descriptors by the magnitude of the second Fourier descriptor (i.e. $|S(1)|$ ); thus the scale invariant vector $(\mathrm{S}(\mathrm{k}))$ may be written

$$
\text { as } \mathrm{S}(\mathrm{k})=\frac{S(k)}{|S(1)|}
$$

On the other hand, the rotation invariance may be achieved by keeping the shape of the original contour representable via the rotation invariant Fourier descriptors, the orientation of the basic ellipse is used, which is defined by the Fourier descriptors $s(1)=r_{1} \mathrm{e}^{\mathrm{j}} \varphi_{1}$ and $\mathrm{s}(-1)=\mathrm{r}_{-1} \mathrm{e}^{\mathrm{j}} \varphi_{-1}$, as

$$
T_{e}(n)=s(-1) e^{-j 2 \pi n / N}+s(1) e^{j 2 \pi n / N}
$$

After a number of transformations, equation(8) can be written as

$$
\mathrm{T}_{\mathrm{e}}(\mathrm{n})=\mathrm{e}^{\mathrm{j}\left(\varphi_{-1}+\varphi_{1}\right) / 2}\left[\mathrm{r}_{-1} \mathrm{e}^{-\mathrm{j}(\hat{\varphi}+2 \pi \mathrm{n} / \mathrm{N})}+\mathrm{r}_{1} \mathrm{e}^{\mathrm{j}(\hat{\varphi}+2 \pi \mathrm{n} / \mathrm{N})}\right]
$$

Where $\hat{\varphi}=\left(\varphi_{1}-\varphi_{-1}{ }^{\prime} / 2\right)$.

Equation (9) shows the rotation $\varphi_{\mathrm{e}}$ of the basic ellipse by

$$
\varphi_{\mathrm{e}}=\left(\varphi_{-1+} \varphi_{1}\right) / 2
$$

An ambiguity of $\pi$ radians can be observed by using the orientation of the basic ellipse. Thus the rotation invariant Fourier descriptors are only rotation invariant modulo a rotation by $\pi$ radians.

Moreover, The position of the starting point may be normalised by subtracting the phase of the second Fourier descriptor weighted by $\mathrm{k}$ from the phase of all Fourier descriptors, i.e.

$$
\mathrm{S}(\mathrm{k})=\mathrm{S}(\mathrm{k}) \mathrm{e}^{-\mathrm{j}} \varphi_{1} \mathrm{k}
$$

\subsection{Center of Mass Detection}

A normalized point or center of mass is needed that will be significant point in terms of the contour points of that subject. A rectangle which encloses only the contour of the subject, called bounding rectangle is considered to crop the image according to the perimeter and resized to a fixed height while preserving the aspect ratio (i.e., ratio of silhouette width to its height) using bilinear interpolation.

The resized contour is then copied to a destination image of fixed size by coinciding its centre-of-mass with the centre of the destination image to make it translation invariant.

A 2D Cartesian moment is a gross characteristic of the contour computed by integrating over all of the pixels of the contour. The $(r, s)$ moment of a contour can be defined as

$$
\mathrm{m}_{\mathrm{r}, \mathrm{s}}=\sum_{i=1}^{n} I(x, y) x^{r} y^{s}
$$

Here $\mathrm{r}$ is the $x$-order and $\mathrm{s}$ is the $y$-order, whereby order means the power to which the corresponding component is taken in the sum. The 2D Cartesian coordinate of center of mass $C\left(x_{c}, y_{c}\right)$ is the ratio of the $1^{\text {st }}$ order and $0^{\text {th }}$ order of contour moment [12].

$$
\mathrm{x}_{\mathrm{c}}=\mathrm{m}_{10} / \mathrm{m}_{00}, \quad \mathrm{y}_{\mathrm{c}}=\mathrm{m}_{01} / \mathrm{m}_{00}
$$

$\mathrm{m}_{00}$ moment is the length in pixels of the contours when both $\mathrm{r}$ and $\mathrm{s}$ both are zero.

$\mathrm{m}_{10}$ and $\mathrm{m}_{01}$ denotes moment of $\mathrm{x}$ and $\mathrm{y}$ component. 


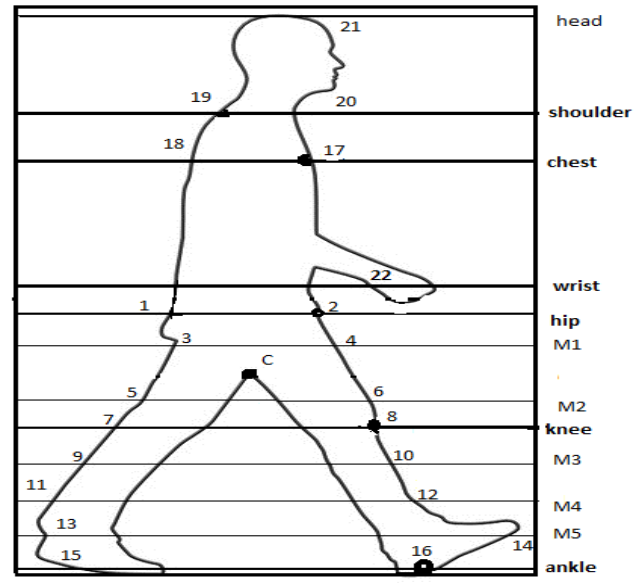

Fig.2. Control point Selection

\subsection{Control point selection}

Selection of control points is very important and imperative in our work, since these points signify the gait characteristics of a subject under surveillance, to be extracted. Anatomical landmarks have been considered on the different locations of the human body as a fraction of subject's height $(\mathrm{H})$. From the concept of anatomical studies [13] the positions of ankle (Ak), knee (kn), Wrist(Wr), hip (Hp), chest (Ch), shoulder (Sl) and head (Hd) are estimated vertically as a fraction of the body height (measured as vertical height of the bounding rectangle enclosing the subject's silhouette contour) as $0.039 \mathrm{H}, 0.285 \mathrm{H}, 0.485 \mathrm{H}, 0.530 \mathrm{H}, 0.720 \mathrm{H}, 0.818 \mathrm{H}$ and $0.870 \mathrm{H}$ measured from the bottom of the subject i.e. maximum y coordinate level. The control points of the contour have been considered from these points, are treated as anatomical landmarks.

Now to have all the control points with the help of anatomical landmark points the following algorithm has been illustrated.

\section{Algorithm 1:}

Input: The boundary point set BP of the subject in the bounding rectangle.

Output: Generate the control points 1-21.

i) The boundary point set $\mathrm{BP}=\left\{\mathrm{b}_{\mathrm{k}}\right\}$, $\mathrm{k}$ is number of boundary points. Each $b_{k}$ has $x$ and y coordinate named $\mathrm{x}_{\mathrm{k}}, \mathrm{y}_{\mathrm{k}}$.

ii) Perpendicular distances $\forall \mathrm{b}_{\mathrm{k}}\left(\mathrm{x}_{\mathrm{k}}, \mathrm{y}_{\mathrm{k}}\right)$ from maximum y coordinate (say $\mathrm{y}_{\mathrm{m}}$ ) (bottom level) has been found out. Let it be $\mathrm{D}_{\mathrm{k}}$,

$$
\mathrm{D}_{\mathrm{k}}=\left\{\mathrm{y}_{\mathrm{k}}-\mathrm{y}_{\mathrm{m}} \mid \forall \mathrm{k}\right\}
$$

iii) The perpendicular distances of anatomical points $\mathrm{Ak}$, $\mathrm{Kn}, \mathrm{Hp}, \mathrm{Wr}, \mathrm{Ch}, \mathrm{Sl}$, Hd from $\mathrm{y}_{\mathrm{m}}$ as $\mathrm{d}_{\mathrm{Ak}}, \mathrm{d}_{\mathrm{Kn}}, \mathrm{d}_{\mathrm{Hp}}, \mathrm{d}_{\mathrm{wr}}$, $\mathrm{d}_{\mathrm{Ch}}, \mathrm{d}_{\mathrm{Sl}}, \mathrm{d}_{\mathrm{Hd}}$ have been calculated as the vertical height of the bounding rectangle.

iv) Comparing these distances $\left(\mathrm{d}_{\mathrm{Ak}}, \mathrm{d}_{\mathrm{Kn}}, \mathrm{d}_{\mathrm{Hp}}, \mathrm{d}_{\mathrm{Ch}}, \mathrm{d}_{\mathrm{Sl}}, \mathrm{d}_{\mathrm{Hd}}\right)$ with $D_{k}$ set, the index points have been found out.

v) These index points are compared with $\mathrm{BP}_{\mathrm{i}}$ (index of boundary point set $)(1 \leq \mathrm{i} \leq \mathrm{k})$
From the matched index value of $\mathrm{BP}_{\mathrm{i}}$ 's the corresponding $\mathrm{x}$ and $\mathrm{y}$ coordinates of corresponding anatomical points are extracted.

vi) From one anatomical landmark point the opposite coordinate can be found out from the set BP. (e.g. the point on hip marked as 2 and the opposite is marked as 1). From a landmark, comparing the y coordinate point with all corresponding $\mathrm{x}$ coordinate points from the set $\mathrm{BP}$, the minimum $\mathrm{x}$ coordinate point will be taken from the set $\mathrm{BP}$, this $(\mathrm{x}, \mathrm{y})$ point denotes opposite point. Similar way points $7,15,18,19$ have been found out.

The middle y coordinate points between centre of mass and hip (say M1), knee and center of mass (say M2), knee and ankle (say M4), knee and M4 (say M3), ankle and M4 (say M5) have been considered and corresponding $\mathrm{x}$ coordinate value have been extracted from the set BP and from the step (vi) the opposite points are also obtained. In this way all the control points (1-22) have been found out and kept in set named CntP serially.

\subsection{Leg Rise from Ground (LRFG)}

From the silhouette images the control points thus extracted as described in the algorithm in section 2.4 , so to get more information about the images in the sequences in feature extraction, the measure, LRFG has to be determined. Maximum y co-ordinate point of the bounding rectangle is marked as the ground level. Now, using the index of this point from the set BP, the corresponding $\mathrm{x}$ co-ordinate is determined. The vertical positions of knee $(\mathrm{Kn})$ are estimated as described by the section 2.4. The height is calculated by the Euclidean distance between head point and the perpendicular point B (shown in Fig.3). The algorithm for finding the leg rise is given below:

\section{Algorithm 2: Calculation of LRFG measure.}

Input: Boundary points set BP.

Output: Maximum normalized leg rise distance from horizontal line of the bounding rectangle.

Step1. A horizontal line which is nothing but the bottom level of the bounding rectangle has been considered.

Step2. Minimum x Co-ordinate has been found out from the boundary point set BP by

$$
\mathrm{X}_{\min }=\min (\mathrm{x})
$$

Where min denotes minimum value and the corresponding y coordinate has been taken from BP and this point denotes the heel point (A).

Step3. Perpendicular line has been drawn from A to C ( $\mathrm{C}$ is the point considered by maximum y coordinate and minimum $x$ coordinate respectively). The distance from the heel point $\mathrm{A}$ to the perpendicular point $\mathrm{C}$ is denoted by PER

Step4. Since the minimum y coordinate point has been treated as head point, corresponding $\mathrm{x}$ coordinated has 
been found out from the set BP. Draw a perpendicular line from head point to the horizontal line point B. Find the Euclidean distance from the point $\mathrm{A}$ to point $\mathrm{B}$, denoted by AB.

Step5. Check whether the distances $A B$ and $A C$ is less than the distance between knee point $(\mathrm{k})$ to Horizontal line, if so, it will be treated as a valid LRFG distance.

Step6. Normalized leg rise (LRFG) is considered by finding the ratio of (PER: AB).

Step7. The maximum normalized leg rise distance among all the stances in a sequence will be taken into the dataset.

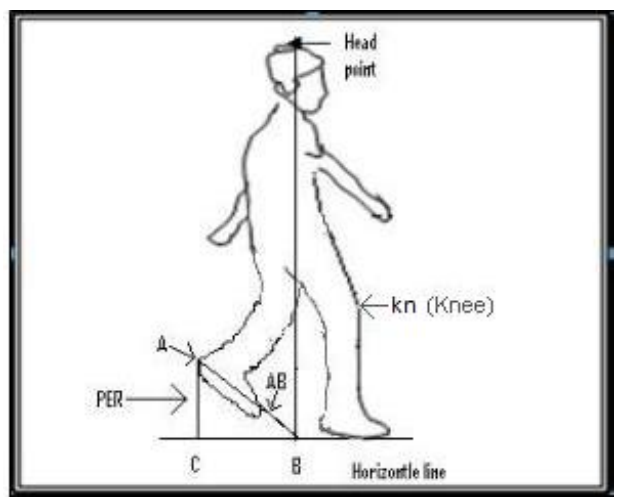

Fig.3. Leg Rise From Ground (LRFG).

\subsection{Distance between Centre of Mass to Control points $(D C C)$}

From the silhouette boundary images the anatomical landmark points or control points have been found out as described in the section 2.4. The centre of mass $\left(\mathrm{C}\left(\mathrm{x}_{\mathrm{c}}, \mathrm{y}_{\mathrm{c}}\right)\right)$ of the object has been described in section 2.3 in equation (13) has been calculated. Now, the Euclidean distances from the centre of mass to the anatomical points of the objects are measured. The distances have been calculated considering the points $\mathrm{C}-2, \mathrm{C}-3, \mathrm{C}-6, \mathrm{C}-$ 7,C-9,C-12,C-13,C-16,C-17,C-19,C-21which is shown by the figure below:

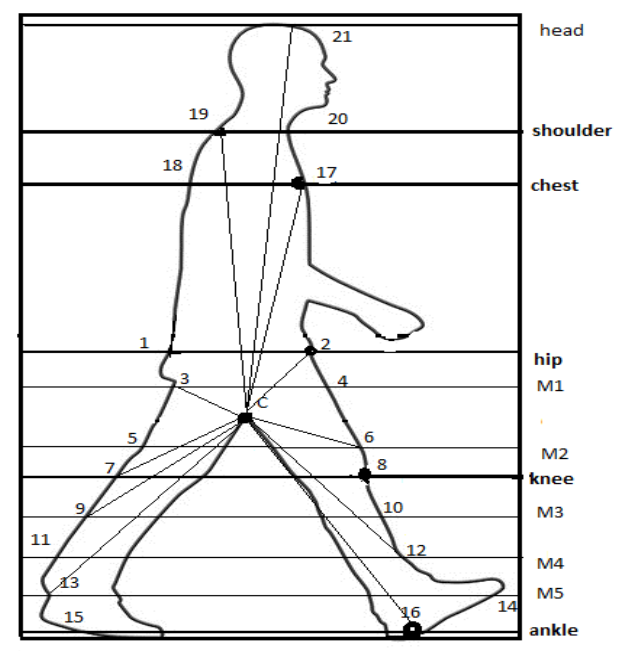

Fig.4. Distance from Centre of Mass to Control Points (DCC)

\subsection{Angles created between the legs with the Centre of Mass (ABLC)}

Another measure ABLC which finds out the angles created between the control points with the centre of mass. The angles between the control points with the centre of mass on the lower half of the centre of mass can be determined by [19] and the following angle have been calculated $\angle \mathrm{Cnt}_{7} \mathrm{C} \mathrm{Cnt}_{6}, \angle \mathrm{Cnt}_{5} \mathrm{C} \mathrm{Cnt}_{8}, \angle \mathrm{Cnt}_{10} \mathrm{C}$ $\mathrm{Cnt}_{11}, \angle \mathrm{Cnt}_{12} \mathrm{C} \mathrm{Cnt}_{13}, \angle \mathrm{Cnt}_{14} \mathrm{C} \mathrm{Cnt}_{15}, \angle \mathrm{Cnt}_{5} \mathrm{C}$ $\mathrm{Cnt}_{16}, \angle \mathrm{Cnt}_{8} \mathrm{C} \mathrm{Cnt}_{13}$ For the upper half i.e above $\mathrm{C}$, the angles $\angle \mathrm{Cnt}_{2} \mathrm{C} \mathrm{Cnt} 3, \angle \mathrm{Cnt}_{17} \mathrm{C} \mathrm{Cnt}_{19}$ have been found out. From the fig. 5 it can be easily realized.

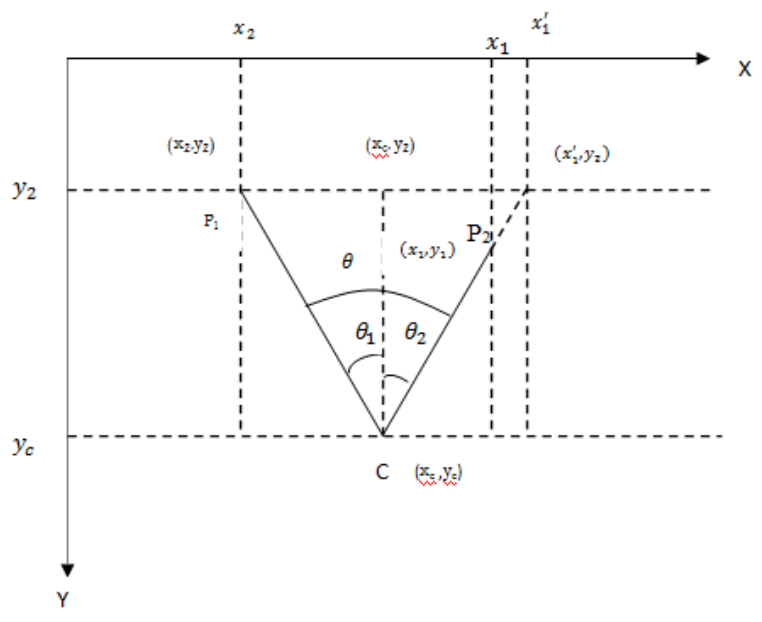

Fig.5. Angles created between control points of upper portion of point $\mathrm{C}$ to the $\mathrm{C}$

2.8 Angles created between the Centre of Mass Knee and Ankle with the (CKA)

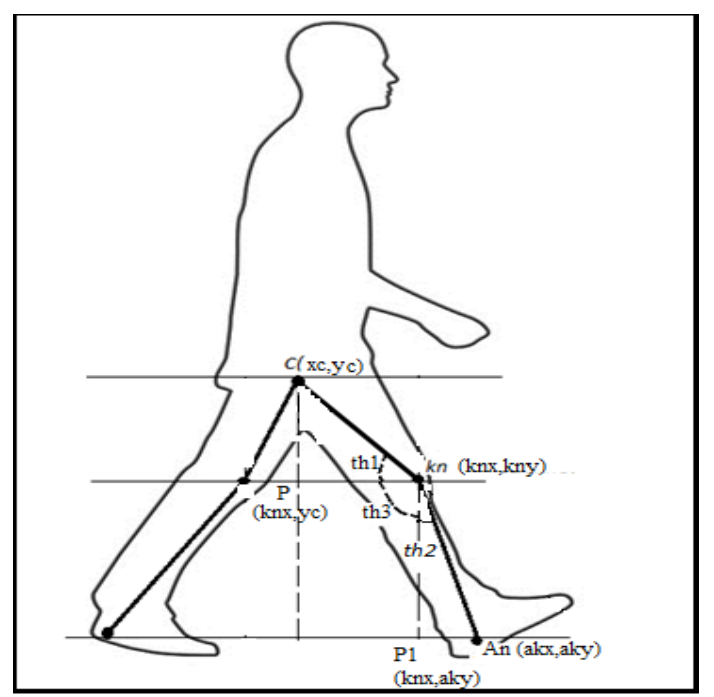

Fig.6. Angles created between the Centre of Mass, Knee and Ankle with the (CKA)

The angle created with centre of mass, leading knee point and leading ankle can be found out. A Perpendicular line is drawn from Centre Of Mass(C) to bottom level and a horizontal line through the knee point $(\mathrm{kn})$. The intersection point between these points is $\mathrm{P}$ 
$(\mathrm{knx}, \mathrm{yc})$. Now the angle created between Centre of mass knee point and $\mathrm{P}$ i.e. $\angle \mathrm{C} \mathrm{kn} \mathrm{p}$

$$
\operatorname{th} 1=\tan ^{-1} \frac{x c-k n x}{y c-k n y} \text {. }
$$

Another Perpendicular line is drawn from knee point to the ground level. Now since this line is perpendicular to the horizontal line via points $\mathrm{P}$ and knee, the angle th3 is obviously $90^{\circ}$. The perpendicular line from knee point to the bottom level intersect the horizontal line drawn via ankle point An at P1 (knx, aky).The angle created between ankle, knee and P1 i.e $\angle$ An kn P1 can be written as

$$
\text { th2 } 2=\tan ^{-1} \frac{a k x-k n x}{k n y-a k y}
$$

Now the angle created between Centre of Mass, knee and ankle can be written as

$$
\text { th }=\text { th } 1+\text { th } 2+\text { th } 3
$$

When the legs will not far away to each other or in case of swing phase the legs are not so stretched, in that case the $\mathrm{x}$ coordinate akx of ankle point will be less than knee point knx.

So, if akx $>\mathrm{knx}$ the angle th can be found out from the equation (17), otherwise i.e. for $\mathrm{akx}<\mathrm{knx}$

$$
\text { th }=\text { th } 1+90^{\circ}-\text { th } 2
$$

\subsection{Angles created between Centre of Mass, Wrist and} knee (CWK)

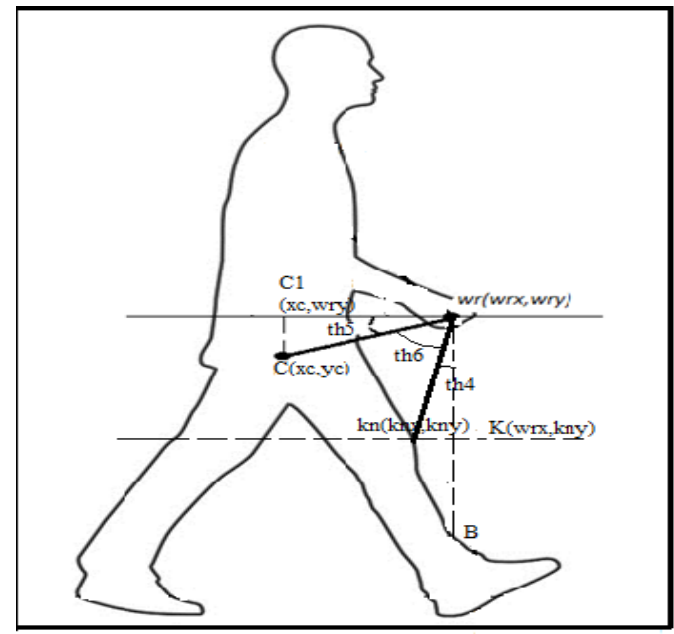

Fig.7. Angles created between Centre of Mass, Wrist and knee (CWK)

To find out the angles between the Centre of mass, Wrist (which is measured from anatomical landmarks by $0.485 \mathrm{H}$ ) and knee point the following procedure is followed. Draw perpendicular line from the wrist to any point on leg (say B) and draw a horizontal line which passes through wrist point wr and the perpendicular line from $\mathrm{C}$ intersect that line at $\mathrm{C} 1(\mathrm{cx}$, wry).Another horizontal line is drawn from knee point which cut the perpendicular line from wr at $\mathrm{K}$ (wrx, kny) Now the $\angle \mathrm{C} 1$ wr $\mathrm{C}$ can be calculated as

$$
\text { th5 } 5=\tan ^{-1} \frac{y c-w r y}{w r x-x c}
$$

the angle $\angle \mathrm{kn}$ wr $\mathrm{K}$ can be calculated as

$$
\text { th4 }=\tan ^{-1} \frac{k n x-w r x}{w r y-k n y}
$$

since $\angle \mathrm{C} 1 \mathrm{wr} \mathrm{K}$ is a right angle the angle $\angle \mathrm{C}$ wr $\mathrm{K}$ can be found out by

$$
\text { th6 }=90^{\circ}-(\text { th } 4+\text { th } 5)
$$

\section{EXPERIMENT AND RESULTS}

The experiment has been conducted by using CASIA$C$ dataset [14]. Here 21 control points have been considered and out of these 11 different angles and 11 different distances and 1 LRFG for each gait sequence or in total 23 parameters have been calculated and treating each column as a parameter value in the extracted feature set. If in a sequence there are $\mathrm{n}$ images then for a person's each gait sequence, the training dataset consists of $23 \mathrm{x}$ n matrices.

\subsection{Mahalanobish Distance}

In this work, it is assumed that these parameters follow Normal Distribution. Let, $X_{1}, X_{2}, \ldots ., X_{23}$ are the random variables corresponding to the parameters. So the joint probability density function (PDF) of these variates is multinormal. Thus, for each sequence, the PDF is Multinormal with different population parameter values, such as population mean vector and population variancecovariance matrix.

For two sample matrices or for two gait sequence of order $23 \times n$ to determine whether they are from same person or not, sample version of Mahalanobis distance [15] has been used.

For sample training and testing vectors, let, $\mu_{1}$ and $\mu_{2}$ be the mean vectors respectively (the mean vectors are vectors of column wise mean values) and $S_{1}$ and $S_{2}$ are the sample variance-covariance matrices of the training and testing data respectively.

Let,

$$
S=\left(S_{1}+S_{2}\right) / 2
$$

Mahalanobis distance statistic says

$$
D^{2}=\left(\mu_{1}-\mu_{2}\right)^{T} S^{-1}\left(\mu_{1}-\mu_{2}\right)
$$


If the value of $D^{2}$ is less than or equal to some threshold value, then it can be inferred that the gait sequence is of same person, otherwise it is not in the database.

\subsection{Threshold value}

For threshold calculation $k$ number of gait sequences of the same person have been considered. In each case, the $D^{2}$ values have been calculated. Let, these $D^{2}$ values are $D^{2}{ }_{1}, D^{2}{ }_{2}, \ldots . ., D_{k}^{2}$.

Now,

$$
D_{1 k}^{2}=\frac{1}{k}\left(D_{1}^{2}+D_{2}^{2}+\ldots . .+D_{k}^{2}\right)
$$

In this way, for $p$ persons and $\mathrm{k}$ sequences each $D^{2}{ }_{1 k}, D^{2}{ }_{2 k}, \ldots . ., D^{2}{ }_{p k}$ have been calculated and an average value is considered i.e.

$$
D_{\text {avg }}^{2}=\frac{1}{p}\left(D^{2}{ }_{1 k}+D_{2 k}^{2}+\ldots . .+D_{p k}^{2}\right)
$$

$D_{\text {avg }}^{2}+\sigma$, has been chosen to be the threshold value where, $\sigma$ is the precision value defined by

$$
\sigma=\frac{1}{p}\left(\left(D_{a v g}^{2}-D_{1 k}^{2}\right)^{2}+\ldots . .+\left(D_{a v g}^{2}-D_{p k}^{2}\right)^{2}\right)^{1 / 2}
$$

In this experiment, gait sequences of 100 persons have been considered and corresponding feature set of gait sequences have been created. The vectors for each person and for each sequence of the order of $23 \mathrm{X}$ 25 (considering in each sequence there are 25 images). Since, 3 sequences of a person have been taken, the feature vector would be in the form of three matrices having dimension $23 \times 25$ and $D^{2}$ values are calculated with sequence 1-2, 1-3 and 2-3 and calculate $D^{2}$ avg and the precision value by applying the equations (25) and (26).

For individual persons the $D^{2}$ values i.e. $D^{2}{ }_{1}, D^{2}{ }_{2}, \ldots . ., D^{2}{ }_{k}$ (where $\mathrm{k}$ is the sequence number, in this case $\mathrm{k}=3$ have been considered) have been calculated. And for the 100 persons the $D^{2}$ avg has been calculated and it is 0.32348 .

\subsection{Training and Testing Algorithm}

\section{Algorithm-3: Training}

Input: Image sequences of different persons.

Output: Training dataset and threshold.

Step 1. Create Database of Silhouette images of different persons.

Step 2. Step2-for each pair wise gait sequence of the same person do the following

a. Find out the boundary of each subject in each image using Fourier Descriptor. b. From the concept of analytical landmarks find out the control points by algorithm 1 .

c. Calculate the Centre Of Mass (C) as described in section 2.3.

d. Create the feature set by following components-

i) The Leg Rise from the ground (LRFG).

ii) The Euclidean Distance between the Centre of Mass and the control points (ABLC).

iii) The Control angle between the Centre of Mass and the control points (DCC).

iv) angles created between the Centre of Mass, Knee and Ankle with the (CKA),

v) angles created between Centre of Mass, Wrist, knee (CWK),

e. Compute the pair wise Mahalanobish-distance for the gait sequences of the same person and store these values for each person.

f. Compute threshold value using the procedure described in section 3.2

\section{Algorithm-4: Testing \\ Input: Unknown Gait Sequence \\ Output: Match or No Match}

Step 3. Take a gait sequence of an unknown person.

Step 4. Extract features by the procedure described in step2- (a)-(d) of algorithm-3.

Step 5. Compute Mahalanobish-distance between the testing data and each training sequence data with predefined threshold value to test for matching.

The precision value is obtained experimentally. A good response of recognition depends on this precision value. This value can be positive or negative. It has been depicted in the figure- 8 . The response of the false rejection rate (FRR), false acceptance rate (FAR) and the correct recognition rate (CRR) in accordance with value of $\pm \sigma$. It has been found out that correct recognition rate was $91 \%$ in our old method but in this proposed approach the CCR is $92.3 \%$ and both the FAR and FRR is $8 \%$ if $\sigma=0.17$.i.e for the threshold value $D^{2} a v g+\sigma=0.49348 \approx 0.5$

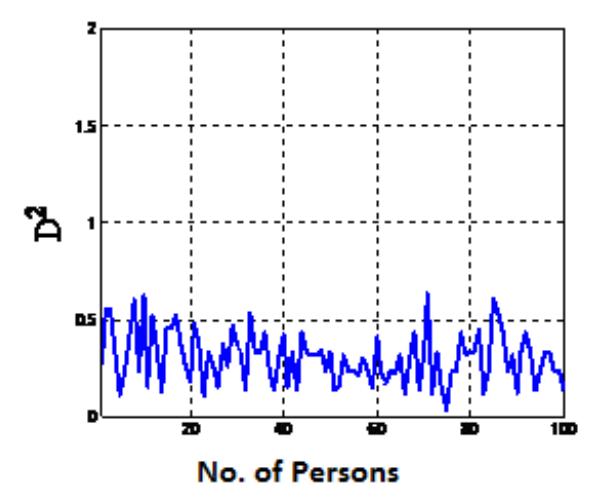

Fig.7. The $D^{2}{ }_{1 k}, D^{2}{ }_{2 k}, \ldots \ldots, D^{2}{ }_{p k}$ values corresponding to 100 persons are mapped in this figure 


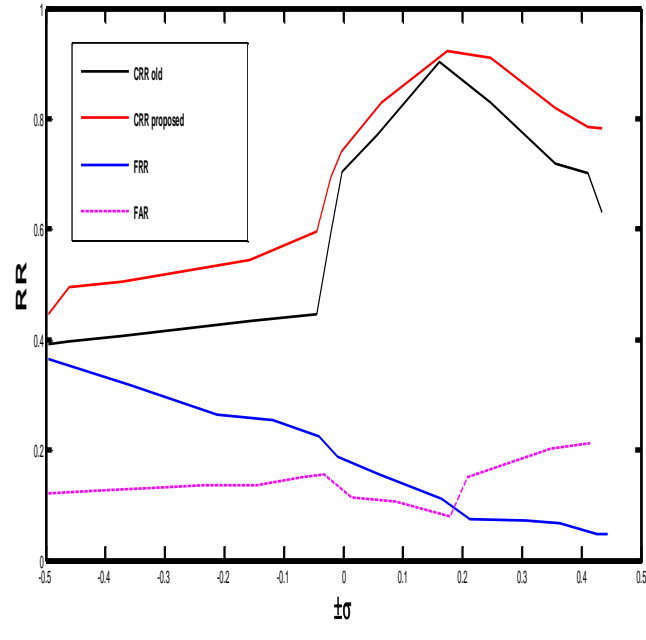

Fig.8. Plot with $\pm \sigma$ and the Recognition Rate

The fig. 8 shows the recognition rates for both the +ve and - ve values of $\sigma$. It shows below the zero value of $\sigma$ i.e. in the -ve direction the response of CRR and FRR, FAR is not appreciable compared to the +ve direction and when $\sigma=+0.17, \mathrm{CRR}$ becomes maximum i.e. $92.3 \%$ and considering the trade of between FAR; FRR the value can be taken at 0.079 of RR i.e.7.9\%. Comparing the two CRR curves i.e. black and red (black shows our old method [19] and red shows proposed approach), it can be seen that CRR of our proposed approach gives us better correct recognition rate.

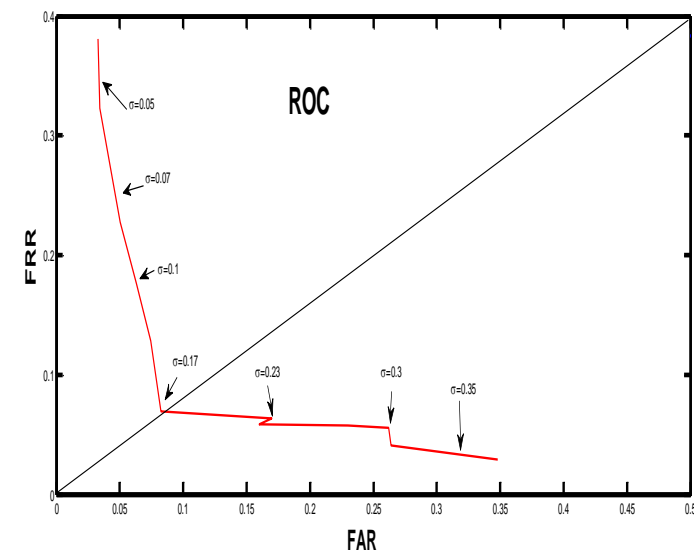

Fig.9. From the ROC curve the ERR value is 0.79 for $\sigma=0.17$ i.e. at this point $F A R=F R R$.

Fig 9 shows ROC for different value of $\sigma$ but when $\sigma=0.17$ maximum recognition rate is obtained.

Table 1. Different Recognition Parameters

\begin{tabular}{|l|l|l|l|l|}
\hline Person & $\begin{array}{l}\text { Correct } \\
\text { recognition }\end{array}$ & $\begin{array}{l}\text { Correct } \\
\text { rejection } \\
\text { rate }\end{array}$ & $\begin{array}{l}\text { False } \\
\text { acceptance } \\
\text { rate }\end{array}$ & $\begin{array}{l}\text { False } \\
\text { rejection } \\
\text { rate }\end{array}$ \\
\hline 100 & $92.3 \%$ & $92 \%$ & $7.9 \%$ & $8 \%$ \\
\hline
\end{tabular}

To examine the performance of the proposed algorithm, comparative experiments have been conducted which is shown in table-2. From Table-2, it is clear that the method, proposed here, has shown significant improvement in performance as compared to other recent methods for same database.

Table 2. Comparison of different approaches with our methods

\begin{tabular}{|l|l|l|}
\hline Method & Database & Recognition Rate \\
\hline $\begin{array}{l}\mathrm{M}_{\mathrm{G}}^{\mathrm{ij}}(x, y)+A C D A \\
{[16]}\end{array}$ & CASIA-C & $34.7 \%$ \\
\hline GEI [17] & CASIA-C & $74 \%$ \\
\hline 2DLPP[18] & CASIA-C & $88.9 \%$ \\
\hline $\begin{array}{l}\text { Our Old method } \\
{[19]}\end{array}$ & CASIA-C & $91 \%$ \\
\hline Proposed Method & CASIA-C & $92.3 \%$ \\
\hline
\end{tabular}

\section{CONCLUSION}

This paper focuses on the idea of using Fourier descriptor based shape extraction on silhouette-based gait characteristics. Gait has a rich potential as a biometric for recognition though it is sensitive to various covariate conditions, which are circumstantial and physical conditions that can affect either gait itself or the extracted gait features. Example of these conditions includes carrying condition like backpack, briefcase, handbag, etc., view angle, speed and shoe-wear type etc. or physical conditions like pregnancy, injury on legs etc. Our technique is very good in terms recognition accuracy but here only lateral view with movement been considered, so there is a good scope to extend this technique on different view angle between camera and object.

\section{ACKNOWLEDGEMENT}

The authors would like to thank Mr. Hongyu Liang for providing the database of the silhouettes named "CASIA Human Gait Database" collected by Institute of Automation, Chinese Academy Of sciences. Their thanks also go to those who have contributed to the establishment of the CASIA database [14].

This work has been carried out at "Center for Machine Learning and Intelligence" under computer science and engineering department of Supreme Knowledge Foundation Group of Institutions (SKFGI), Mankundu, Hooghly, West Bengal, India.

\section{REFERENCE}

[1] Giovanni, B.: 'On the movement of animals', SpringerVerlag, 1989.

[2] Braune,W., Fischer, O.: Translated by Maquet ,P., Furlong, R.: 'The human gait (Der gang des menschen) '. Berlin/New York: Springer Verlag, 1987.

[3] Murray, M., Drought, A., and Kory, R.:'Walking Pattern of Normal Men', Journal of Bone and Joint Surgery, 1964, vol. 46-A, no. 2, pp. 335-360.

[4] Jiwen Lu, Erhu Zhang, "Gait recognition for human identification based on ICA and fuzzy SVM through multiple views fusion", Pattern Recognition Letters, Vol. 28, pp. 2401-2411, 2007.

[5] L. Lee, W.E.L. Grimson, Gait analysis for recognition and classification, in: Proceedings of the International Conference on Automatic Face and Gesture Recognition, 2002, pp. 155-162. 
[6] D. Cunado, M. Nixon, and J. Carter, "Automatic Extraction and Description of Human Gait Models for Recognition Purposes," Computer Vision and Image Understanding, vol. 90, no. 1, pp. 1-41, 2003.

[7] Gavrila, D.:' The Visual Analysis of Human Movement: A Survey', Computer Vision and Image Understanding, 1999, vol. 73, no. 1, pp. 82-98.

[8] Wang, L., Hu, W.M., and Tan, T.N.: 'Recent Developments in Human Motion Analysis', Pattern Recognition, 2003, vol. 36, no. 3, pp. 585-601.

[9] Rousseeuw, P. J.: 'Least Median of Squares Regression' Journal of the American Statistical Association December 1984, Volume 79, Number 388.

[10] Kuno, Y., Watanabe, T., Shimosakoda, Y., and Nakagawa, S.: 'Automated Detection of Human for Visual Surveillance System,' Proc. Int'l Conf. Pattern Recognition, 1996, pp. 865-869,.

[11] A. K. Jain. Fundamentals of Digital Image Processing. Information and Systems Science Series. Prentice Hall, 1989.

[12] M.S. Nixon, A.S. Aguado, Feature Extraction and image Processing, 2nd ed.., Elsevier, London, 2006.

[13] Winter, D.A.: 'Biomechanics and Motor Control of Human Movement, third ed., John Wiley \& Sons, New Jersey, 2004.

[14] CASIA gait database http: // www. sinobiometrics .com.

[15] Mahalanobis, P. C.: 'On the generalised distance in statistics'. Proceedings of the National Institute of Sciences of India 2 (1): Retrieved 2012-05-03, 1936, pp. 49-55.

[16] Bashir, K., Xiang, T., Gong, S.: 'Gait recognition without subject cooperation', Elsevier Pattern Recognition Letters 2010.

[17] Jungling, K., Arens, M.: 'A multi-staged system for efficient visual person reidentification' MVA2011 IAPR Conference on Machine Vision Applications, June 13-15, 2011, Nara, JAPAN

[18] Zhang, E., Zhao, Y., Xiong, W.: 'Active energy image plus 2DLPP for gait recognition'. Signal Process. 2010, vol- 90 , pp. 2295-2302.
[19] Ghosh, M., Bhattacharjee, D.: 'An Efficient Characterization of Gait for Human identification', I.J. Image, Graphics and Signal Processing, 2014, 7, 19-27

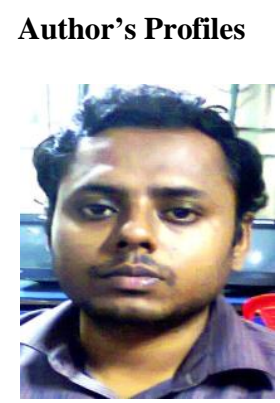

Mridul Ghosh born in October 29th, 1982 and received his B.Sc (Hons.) degree in Physics from Calcutta University, Calcutta, India; B.Tech and M.Tech degree in computer science \& Engineering from University college of science \& Technology, Calcutta University in the year of 2003, 2006 \& 2008 respectively. $\mathrm{He}$ is currently working towards his Ph.D. (Eng.) award from Jadavpur University, Kolkata, India. His current research interest include pattern Recognition, Image processing, Fuzzy Logic.

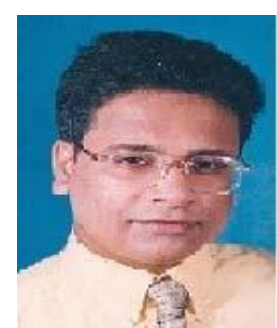

Debotosh Bhattacharjee received the MCSE and Ph. D. (Eng.) degrees from Jadavpur University, India, in 1997 and 2004 respectively. He was associated with different institutes in various capacities until March 2007. After that he joined his Alma Mater, Jadavpur University. His research interests pertain to the applications of computational intelligence techniques like Fuzzy logic, Artificial Neural Network, Genetic Algorithm, Rough Set Theory, Cellular Automata etc. in Face Recognition, OCR, and Information Security. He is a life member of Indian Society for Technical Education (ISTE, New Delhi), Indian Unit for Pattern Recognition and Artificial Intelligence (IUPRAI), and senior member of IEEE (USA).

How to cite this paper: Mridul Ghosh, Debotosh Bhattacharjee,"Gait Recognition for Human Identification Using Fourier Descriptor and Anatomical Landmarks", IJIGSP, vol.7, no.2, pp.30-38, 2015.DOI: 10.5815/ijigsp.2015.02.05 